\title{
Born of geographical environment, coloring for regional context: Concept and progress of geo-architecture
}

\author{
WANG Fang \\ Sino-German Joint Laboratory on Urbanization and Locality Research (UAL), College of Architecture and \\ Landscape Architecture, Peking University, Beijing 100871, China
}

\begin{abstract}
In today's world, where re-examination has been given to cultural globalization, the relationship between architectures and specific regions is one of many specific issues. At this time, the study of architecture from the perspective of geography has significant meaning for treasuring regional characteristics and protecting the essence of ethnic culture. The study of geo-architecture includes two aspects: the influence of geographical environment upon architecture and reflexively, architectural responses to geographical environment. In "Geo-Architecture and Landscape in China's Geographic and Historic Context Series" (4 volumes), published by Springer Nature in 2016, the contents of "geo-architecture" has been interpreted from various angles as form, function, semantics and symbol. This book is the first to be entitled "Geo-Architecture", which studies the relationship between geography and architecture from the perspective of geographical environment and historical context in China. The book's research reveals the influences of different geographical scales, such as zone (macro), region (middle) and site (micro) scales, on architecture; it discusses dynamic and systematic thought, including such research elements as "Object-Subject-Time", and it reflects views on pattern and process of cultural landscape. Research on geo-architecture aims to help construct the security pattern of the cultural landscape for the new requirements of a new era, which is used to optimize spacial structure, stimulate heritage conservation and renewal, respect the geographical environment, pay attention to historical culture and sustain emotional memory.
\end{abstract}

Keywords: geo-architecture; geographical environment; cultural landscape; Springer Nature

\section{Introduction}

The geographical environment is the foundation of human survival. The natural and social environments constitute the condition for mankind's survival, and both of them integrate and influence each other. As the physical space form resulting from human activities on the natural landscape, architecture is the fruit of mutual influence and interaction between hu-

Received: 2016-10-08 Accepted: 2016-12-09

Foundation: Joint Sino-German Research Project supported by the Sino-German Center (NSFC and DFG), No.GZ1201

Author: Wang Fang (1973-), PhD and Professor, specialized in cultural landscape and its locality of built environment in urban and rural areas. E-mail: wfphd@pku.edu.cn 
man culture and natural environment and of the complex of natural and human factors. In today's world, where we have reexamined cultural globalization, the relationship between architecture and specific regions belongs to one specific issue. The study of architecture from the perspective of geography is of some significance in treasuring regional characteristics and protecting the essence of ethnic culture.

With the development of the discipline and the rise of new requirements, geography places tremendous emphasis on the study of the relationship between society and the natural environment and on the exploration of earth's surface laws. As the product of economy, politics and society at certain historical periods, architecture comes into being because of intentional and purposeful activities, which could reflect geographical features and the diversity resulting from the combined action of the human and natural environments. Research into geo-architecture corresponds to the major topic of how to coordinate the "man-land relationship" in the fields of geography and architecture.

The study of architecture is perceptual and inevitably rational. For example, Aldo Rossi (1984) brought architecture into the study of the city as a rational theoretical system, and spoke about the influence of such basic powers on the architecture of urban development as history, collective wills, economics, society and politics, which was at the forefront of research into "the architecture of the city". By taking the geographical environment as the mother of architecture's inoculation, survival and evolution, geo-architecture studies the architectural representation and development process by employing the perspective, thought and method of geography, especially natural geography, human geography, and historical geography. In this study, knowledge of natural geography includes such aspects as geology, landform, climate, hydrology, vegetation, and so on, for example, typical landscapes such as the Gobi desert, Tibetan Plateau, and loess landforms; and that of human geography concerns, for example, religion and ethics, manners and customs, economics and politics; and that of historical geography concerns, for example, human migration, regime changes, and foreign influences.

In the concept of geo-architecture, the architecture is not the protagonist; instead, the relationship between architecture and geographical environment is the core, reflecting the influence of geographical environment upon architecture and reflexively, architecture responses to the geographical environment. As an interdisciplinary research fruit combining the two disciplines of architecture and geography, geo-architecture is a study focused on the interactive relationship among architecture, environment and time based on the understanding of "man-land relationship".

At the same time, geo-architecture belongs to the study and scope of cultural landscape. In the constantly changing and developing society, the built environment in urban and rural areas always faces challenges and opportunities. Conservation and renewal are a lasting hot issue. The concept of conservation has developed from the protection of a single building to the protection of the natural and cultural environment to which that building belongs. However, in the process of development, conservation is usually under appreciated and must give way to city construction. In particular, during the process of rapid urbanization and great transformation, when the urbanization rate in China has surpassed 50\%, cities are faced with even more problems and challenges, such as land use, transportation, serious pollution, etc. In the cultural field of city construction, because of the lack of cultural connotation and re- 
gional features, there are problems involving thousands of cities showing the same images and dismantling authenticity to build fake ones. The traditions, ethics, and regional cultural landscapes in some urban and rural areas are facing such dangers as destruction, mutation and disappearance. In the presence of the ever-increasing and severe man-land relationship crisis, the Chinese government underlined the significance of ecological civilization and the concept of sustainable development that emphasizes the harmonious co-existence of human and nature. "Geo-architectural" research, carried out under these circumstances, is of great significance. In the meantime, the research ideology of geo-architecture is universal, which could provide a reference point for analyzing the cultural landscape in other countries. Human civilizations have left behind many bright and dazzling architectural legacies around the world which reveal the profound understandings of people from different ethical beliefs and areas in the geographical environment.

\section{Organization and content of the series}

In 2016, the author has published Geo-Architecture and Landscape in China's Geographic and Historic Context Series" (4 volumes) with Springer Nature, a world-renown science and technology publisher. The series is the very first to be entitled "Geo-Architecture," which studies the relationship between geography and architecture from the perspective of geographical environment and historical context in China.

This kind of writing has a few precedents in the field: The Language of Post-Modern Architecture (1977) and The Garden of Cosmic Speculation (2003) by Charles Jencks may be the closest, but he writes purely from an Anglo-centric viewpoint. About China's vast architectural heritage within the context of its own tradition, Chinese Architecture (2011) by Yanxin Cai, Chinese Architecture (2002) by Xinian Fu et al., and House Home Family: Living and Being Chinese (2005) by Ronald Knapp and Kaiyin Lo could be mentioned. These works provide the historical, socio-political context necessary to begin to understand China's built form. In the words of Associate Professor Annette M. Kim, in the Department of Urban Studies and Planning at MIT, "What is novel about the series is that she further extends this intellectual project of looking at Chinese architecture through Chinese eyes, by taking it one provocative step further" (Wang, 2016a, b, c, d: back cover). Rooted in the profound theory of geography and the colorful practice of architecture, research on geo-architecture reflects a kind of dialogue between human activity and geographical environment. In the long history of mankind, much classical architecture has been left behind that complies with, echoes or improves its geographical environment. From the perspective of geographical features, regional context, and historical evolution, this work searches for the inner connection between architecture and geographical environment.

On selecting research objects for geo-architecture, the range and scale of "architecture" is expanded, not only to include the traditional single building or building cluster but also excellent artificial large-scale constructions reflecting human wisdom, which are a splendid mark left on the earth's surface. Some research objects on which the architecture field focuses less include the Mani field showing worship, the ancient postal road connecting trade and transportation, the tree-embracing pagoda born under the joint effect of climate conditions and religion customs, and some artificial works marking specific geographical position 
concepts such as the Lingqu Canal, which connects the Xiangjiang River and Lijiang River as a watershed area, and the Gaocheng Astronomical Observatory, indicating the "earth's core". Everything mentioned above are splendid phenomena embodying human wisdom.

In the series, 103 cases are selected out of 300 by the author and teammates through the scientific method and process, and they are distributed nearly all over the provinces of China, including autonomous regions, municipalities and special administrative regions. The cases have something in common, which demonstrates their close connections with a typical geographical environment. To obtain first-hand materials, the research team made great efforts to travel to conduct site surveys. Thus, over $90 \%$ of the cases featured in this series were visited, experienced and scrutinized first-hand by research team members.

The series tries to explain its profound content in simple terms with texts and over 1000 pictures. Composed of 4 volumes in ten independent and mutually connected parts, the series interprets geo-architecture from four perspectives: form, function, semantics and symbol. In the organization of each case, there is a "Key Geographical Concept" used as the guide to interpreting the case in the beginning and analyses of geomorphological features, climatic features, cultural features, and vegetation features where the buildings are. The series is based on interdisciplinary research findings so that the unintelligible and important knowledge points can be elucidated. For this purpose, there is a section entitled "Extended Reading" in the main body and a "Geographical Interpretation" in the end section. While analyzing architecture, the series also interprets and encompasses the colorful human culture, nature and historical geography in China, to help readers understand the interrelationships between buildings and specific geographical conditions.

(1) Volume 1: Geo-Architecture Wandering in the Landscape

Volume 1 mainly focuses on "form," including three parts and 27 cases. Part one is "Conversation and Sentiment". "Conversation" discusses collisions between architecture and geography, architecture and humanity, and between architecture and architecture, while "Sentiment" reflects the ancient Chinese notion of emotional relief through nature, expression of emotion through the landscape. Both of them focus upon the interaction of architecture and its environment. Part two is "Halting and Advancing", which comprise two major topics - important transportation and scenic routes, as well as related artificial constructions such as pavilions, towers, and clan halls within the concept of geo-architecture. Part three is "Integration and Isolation". Faced with different natural and human environments, architectural activity has always been an important method through which human beings are able to remold geography in order to meet their needs for integration and isolation. The forms of geographical integration and isolation expressed through architecture reflect their historical and cultural context.

(2) Volume 2: Geo-Architecture Inhabiting the Universe

Volume 2 mainly focuses on "function", including two parts and 26 cases. Part one is "Houses and Tombs", which are the resting places of human beings, keeping out the wind and rain, as well as external man-made threats. Houses are the homes of the living, called "Yang"1) dwellings in Chinese culture; tombs are the homes of the dead, called "Yin"

1) Yang and yin are a pair of traditional Chinese philosophical concepts that represent the two opposite or contrary principles in nature and how they give rise to each other as they interrelate. They are used in various fields of traditional Chinese culture, including religion, philosophy, the calendar, fengshui. 
dwellings. To some degree these two architectures reveal the wisdom with which different ethnic groups adapted to different geographical environments at different times throughout their long history. Part two is "Religion and Empire". China is not a theocracy. Emperors of different dynasties throughout history worshiped a number of religions, including Buddhism, Taoism, Islam, and Christianity. This connection between imperial power and religion, along with its geographical and cultural connotations, directly impacted the pattern and layout of religious and imperial architecture. In each dynasty, emperors constructed palaces and gardens while believers built temples and monasteries. Religious and imperial architecture form a beautiful horizon of architectural response.

\section{(3) Volume 3: Geo-Architecture Blending into Nature}

Volume 3 mainly focuses on "semantics", including three parts and 28 cases. Part one is "Embedments and Highlights", which are two notions that refer to a pair of contrasting architectural forms in geography. "Embedments" discuss how architecture was born of geography, while "highlights" discuss architecture whose form is based on and finally surpasses a certain geography. The brilliant responses toward local geography are a stunning display of human wisdom. Part two is "Enjoying Mountains and Rejoicing in Waters". Architecture enthusiastically adapts to mountainous and aquatic terrain, often realizing a skillfully contrived utilization of these two resources in the process. Such architecture has become a live exhibit of folk customs and serves to record in profound detail the long history of mankind's recognition of nature. Part three is "The Language of Stone and Earth", which is about realizing architectural response to geography through a creative selection of materials. Stone, earth, bamboo, grass and wood - all materials of natural origin, simple and commonplace have been used in unique ways as local architectural materials in China for thousands of years. This method of selecting building materials reflects the combined impact of the surrounding natural environment, climate conditions and resultant vegetation and hydrology upon the building site. The combined effect is such that the architecture grows out of the surrounding natural and human environment.

\section{(4) Volume 4: Symbolism and the Language of Geo-Architecture}

Volume 4 mainly focuses on "symbol", including two parts and 22 cases. Part 1 is "Implantation and Mixture". Implantation and mixture are two expressions of the way in which Chinese architecture communicates with and assimilates foreign elements. "Implantation" is the architectural expression of one foreign culture in the context of local architecture, and "integration" is that of multiple cultures. Thus, examples of mixture appear in greatest numbers in cities that were once foreign colonies. Part 2 is "Metaphor and Mimicry". Chinese traditional culture is profound and multifaceted and thus has a great influence upon architecture. The relationship between architecture and geography is not merely the embodiment of physical and functional demands but rather a formal expression of the materialization of culture. This explains the vast number of villages, building clusters and individ-

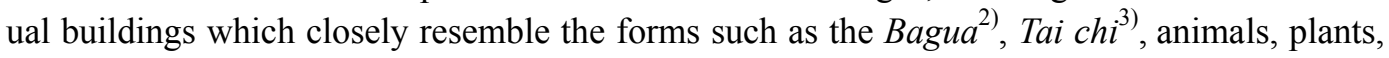

2) Bagua, also named Eight Trigrams, is a basic philosophical concept of ancient China. It is a yin and yang system that can be composed of eight different forms and used to symbolize various natural and human phenomena.

3) Tai chi (Mandarin: tai ji), in Chinese philosophy, reflects the concept of the ultimate source and motive force behind all reality. In the I Ching, tai chi is source and union of the two primary aspects of the cosmos: yang (active) and yin (passive). 
etc. This kind of mimicry creatively places living and working places within the natural geographical environment and, by seeking a relationship between architecture and its surrounding environment, comes to express people's hopes and dreams, evolving slowly to take on certain cultural connotations. This type of geo-architecture is in effect the condensation of human culture into a lively symbolic form.

\section{Major characteristics of the series}

Based on the "influence-response" relationship, this series approaches the concept of "geo-architecture", which includes the three geographical scales of "zone, region and site" and the three research elements of "Object-Subject-Time" (OST), by constructing an analytical framework of pattern and process to reveal the diverse locality characteristics of architectural landscapes, including interactivity with the natural environment, interactions with humans, and change over time.

(1) Geo-architectural research considering different geographical scales

Key research in geography is based on regional differences in and the development principle of the geographical environment. From the perspective of geography, the different space scales, such as zone (macro), region (middle) and site (micro) scales, exert different influences on architecture. Meanwhile, from a geographical angle, the research contents of geography are macroscopic and the crossover study with architecture extends research interests in the micro scale of geography.

The zonality principle is the first basic law in the geographical environment; it determines its regional differences and evolution, including horizontal zonality and vertical zonality. Horizontal zonality includes latitudinal zonality and humidity zonality, while vertical zonality reflects on the regional differentiation principle from the equator to the two poles, with global scale characteristics and results from solar radiation decreasing from low to high latitudes. Humidity zonality abides by the mainland scale regional differentiation law and distributes in a belt pattern from sea to land due to the land-sea distribution; it often parallels the coastline, which also causes the parallel distribution of vegetation and soil to the coastline. Vertical zonality means the laws that the climate, vegetation, soil and natural landscape distribute in a belt pattern according to rising altitude. Because of the reasons mentioned above, world architectures take on colorful presences.

The zonality law exerts its influence through its zone bit, and the geographical boundary is the linearization of zone bit, whereas the region is the manifestation of zone bit on the surface (Hu et al., 2016: 5). As a geographical unit, region reflects a differentiated part of the earth's surface space. The region possesses diversity in classification, such as natural region, political region, economic region and cultural region; the region is of historic significance, and region boundaries are changeable throughout history, as are the spaces included in the region. The natural region changes according to natural laws, of which the changing speed is rather slow. The socio-economic region changes according to social and economic laws, of which the changing speed is faster (Hu et al., 2016: 121-122). The region belongs to the geographical concept of the medium scale. The regional culture and natural features exert an influence on architecture; for example, the southwestern area in China is located in the transitional zone of the Eurasian plate and Indian plate, and because of the 
fierce geological movement, the landform of high mountain and low valley has come into being under the extrusion of those two plates. In this region, there are two kinds of vernacular dwellings with distinctive geographical characteristics: Aba Tibetan Village in Sichuan and Hani Ethnic Village in Yunnan, both of which are located near $112^{\circ}$ east longitude. Because the latitude of Aba Tibetan Village is $10^{\circ}$ northward compared with that of Hani Ethnic Village in Yuanyang, and 1000-2000 meters higher than the latter, under the combined influence of latitude zonality and vertical zonality, Aba Tibetan Village belongs to a plateau climate zone while Hani Ethnic Village is located in the transitional zone of the subtropical monsoon climate area and plateau climate area. Though both of them, with precipitous topographies, are located in the alpine and gorge region of the southwestern part of China, the climates, vegetation and living styles in these two areas differ greatly, and these differences are also demonstrated on their architectures.

The characteristic of a site is formed by the local factors' influences on the most basic and lowest geographic unit. From a nature standpoint, the landscape is an amalgamation of geological and geomorphologic conditions. Once combined with the cultural inheritances and expressions of different eras, regions, ethnic groups, and architecture, it possesses a rich cultural environment and symbolic significance. There is a saying in Chinese that "the wise rejoices in water, the benevolent enjoys mountain", from which we can see that natural landscapes and human landscapes complement each other. From the angle of natural landscapes, the mountain and water reveal such conditions as geology and landform; but when combined with cultural features of different ethicities in different regions and in different ages, mountains and water could possess profound humanistic connotations and symbolic significance. Architectures are important carriers for expressing cultural implications and have many ways of responding to the mountains and water surrounding them, including the ingenious adaption of the traditional dwellings of different regions and ethicities to the mountains and waters to form conditions for comfortable living, such as a terraced field village in a mountainous environment, a stilted house in a waterfront environment and a cave dwelling in a loess hill. It also includes intelligent development and utilization of mountain and water resources, and development of a traditional nationality handicraft industry, to reach professional enthusiasm; examples include traditional villages using hydraulic technology to develop ceramic industry, and a prospect containing mountain and water culture formed by architecture and mountain screen and water shadow, as in Dali's Three Pagodas with the Cangshan Mountain and Erhai Lake forming a unique reflection in the water scenery, or the Badain Jaran Lamasery by a sand hill and a lake in the desert.

(2) Geo-architectural research embodying dynamic and systematic thought

Research on geo-architecture includes the three elements of "Object-Subject-Time". From the perspective of architecture, most research on buildings focuses on the physical space's technology and art, and the originality of the series embodies dynamics and systematic thought, including such aspects as objective elements, subjective cognition, and time cues.

The objective elements of geo-architecture could contain two parts: physical elements, such as cultural relics, architectural scene, spatial patterns, and surroundings, as well as non-physical cultural elements, such as cultural deposits, historical allusions, toponymic changes, and stories and anecdotes. Human is the participant in building behavior and executor in social practice. Subjective evaluation is comprehensive and is based on the cogni- 
tion of objective facts by adding multiple elements such as sole judgment, value orientation, ideology and outside intervention. The study of the time dimension is equivalent to probing into the subjective and objective elements of geo-architecture based on a synchronicity perspective, and to further screen out the information of the special object elements which could represent the development process of the architectures from a diachronic aspect, and present this information in a present and future time dimension.

Therefore, research on geo-architecture reflects its dynamism, continuity and integrity. In terms of dynamism, the interrelation between buildings and geographical environment is not unalterable, but rather a dynamic process in which the architecture and its environment evolve with changes in time. All of the elements have forged dynamic relationships, which could be seen in diachronism and synchronicity in the time dimension. The form and function of architectures have indicated this dynamic relationship, which could reveal elements that are relatively stable, declining or developing. Continuity refers to the meaning connection between architecture and the geographical environment, and indicates architecture's development process. All meaningful architecture has countless closed connections with its environment, which could become the key point for the continuity of city memory, and also a symbol for the changing of city memory. As for integrity, it can be found in the development of geo-architecture as a hidden concept that preserves and develops the historical context and geographical environment, namely, to master the development track of historical culture in the whole meaning of urban and rural space culture, so that it is an integral geographical and historical conservation and development concept.

(3) Geo-architectural research including the consideration of pattern and process

Because geo-architecture is a dynamic concept, it should be interpreted from a perspective of time and space. The classic pattern concept includes geographical region, region type, region structure and space form, and modern geography focuses more on process study. Process study is very important; on one hand, for the interpretation of patterns requires a process study, to explore the interrelationships hidden behind the pattern laws, and the mechanism and structure deciding the pattern form; on the other hand, from typical case to generalization, process research should be carried out combined with a pattern study framework. Pattern is static and process is dynamic. Geo-architecture reveals the historically evolving process of human behavior on space.

In the geographical environment, the generation of new architectures or structures and the renewal of traditional architecture or community should all be regarded as the outcome of development and reproduction. Each region has its own ecological and cultural structure, and development and reproduction are close to the structural characteristics of living organisms. Process is the procedure in which the event or behavior is produced and reproduced or transferred into a system or structure. For the study of "process", there are two typical explanations: (1) process is as the process for space or time, and usually a descriptive narration is adopted in the study; (2) process is as the mechanism and it is explanatory, and tries to explain how and by what means an event occurs. The formation of architectural form has randomness and more certainty. The limitation and stimulation of the external environment has a far-reaching influence on formation.

To summarize, the study of geo-architecture indicates the transplantation and permeation of geographical theory and technological means into the architectural field. Meanwhile, it 
also enriches and expands the research field of geography, which reveals geographical research on a microcosmic space scale; all of these could bring new information into geographical research, to advance the complementarity between the disciplines of geography and architecture.

(4) Peer evaluation

Regarding the research idea of geo-architecture, Professor Liangyong $\mathrm{Wu}$ of Tsinghua University, a Member of the Chinese Academy of Sciences as well as the Chinese Academy of Engineering, wrote an inscription (Wang, 2016a, 2016b, 2016c, 2016d: Inscription) especially for the author:

Admire the great earth,

Impart your emotions to the mountains and the waters.

The rationale of geography,

The thinking of the architect.

Many peers, such as Professor and Chair Rahul Mehrotra of the Department of Urban Planning and Design at the Harvard Graduate School of Design, Assistant Dean Stephen Ervin at the Harvard Graduate School of Design, and Associate Professor Annette M. Kim of the Department of Urban Studies and Planning at MIT, have given high academic evaluations in the "Foreword" and "comments in back cover" as follows:

"This question is however interrogated in the most interesting and rigorous way by introducing the category of geography, which she extends into geo-architecture - a suggestion that architecture and its relationship to a particular geography is also a way to understand the social and cultural contracts that have evolved in that geography.... The collection also sets an important precedent for the examination of traditions in landscape and architectural design for many parts of Asia" (Wang, 2016a, b, c, d: viii). "This unprecedented work will be a unique and valuable contribution to the literature. Even the unique and intriguing title 'Geo-Architecture' is an invention, one that nonetheless resonates, especially once the author has her say"; "One reason why there would be interest is because her research would fill some significant gaps in the literature" (Wang, 2016a, b, c, d: back cover).

\section{Conclusions and future focus}

From the perspective of geography and architecture, research on geo-architecture aims to discuss the inheritance and innovation of local culture and the maintenance and enrichment of regional features. The urbanization that China is undertaking is unique, and there is no referential experience worldwide for us. Therefore, this study is necessary based on local and real problems in China and addresses serious issues, such as deficiency in cultural connotation and regional features of the urban and rural building environment in the urbanization process. Faced with the broken cultural landscape in the urban and rural building environment, efforts must be made to search for the structural network and critical nodes that could exert a stabilizing function in time changes. Attention is also paid to the dialogue and inheritance way between network and nodes. Geo-architecture is a typical research object and a good entry point to help explore the cultural landscape and its locality in the urban and rural built environment. Future research would continue to focus on the following aspects:

1) Persistence: In the comprehensive geographical environment, what key, irreplaceable, 
and continuous pattern elements does geo-architecture show? To a large extent, scientific research into laws explores stable elements and their persistence at the macroscopic level. In geo-architecture, these persistence elements, hidden in the built environment as a complex and huge system, could be preserved permanently and continue to stand the test of time. It will also be used to explain the causal relationship between the geographical environment and human constructing behavior, to predict future development and to optimize the cultural landscape system structure.

2) Resilience: How does geo-architecture ensure its pattern evolution to adapt to the changes of time? Through the theory of evolution, we realize that, similar to all living creatures, in the process of urban and rural development only some of them could survive and continue to exist, digest the sudden or gradual fluctuation influences from the outside environment, and carry out self-reconstruction after interference. Therefore, geo-architecture could survive in the built environment, a complex self-adaptive system, after experiencing all kinds of interferences, and interact with its geographical environment.

3) Predictability: How will geo-architecture develop in the future, and what is the greatest security pattern for the cultural landscape? The harmonious co-existence between human's building behavior and the geographical environment is not accomplished in an action, but continues to optimize itself through repeated mistakes and iterations. The evolution of urban and rural areas is varied. However, by understanding the ever-changing function and form of geo-architecture in the built environmental system, and considering comprehensively both pattern and process, research on geo-architecture aims to help construct the security pattern of the cultural landscape for the new requirements in a new era, which is used to optimizing spacial structure, stimulating heritage conservation and renewal, respecting the geographical environment, paying attention to historical culture and sustaining emotional memory.

\section{References}

Cai Y X, 2011.Chinese Architecture. New York: Cambridge University Press.

Fu X N, Guo D H, Liu X J et al., 2002. Chinese Architecture. New Haven: Yale University Press.

$\mathrm{Hu}$ Z L, Chen Z X, Cui H T, 2016. Overview of Geographical Environment. Beijing: Science Press. (in Chinese)

Jencks C, 1991. The Language of Post-Modern Architecture. New York: Rizzoli.

Jencks C, 2005. The Garden of Cosmic Speculation. London: Frances Lincoln.

Knapp R G, Lo K Y, 2006. House Home Family: Living and Being Chinese. Honolulu: University of Hawaii Press.

Rossi A, 1984. The Architecture of the City. Cambridge: The MIT Press.

Wang F, 2016a. Geo-Architecture Wandering in the Landscape (Geo-Architecture and Landscape in China's Geographic and Historic Context Series Volume 1). Singapore: Springer Nature.

Wang F, 2016b. Geo-Architecture Inhabiting the Universe (Geo-Architecture and Landscape in China's Geographic and Historic Context Series Volume 2). Singapore: Springer Nature.

Wang F, 2016c. Geo-Architecture Blending into Nature (Geo-Architecture and Landscape in China's Geographic and Historic Context Series Volume 3). Singapore: Springer Nature.

Wang F, 2016d. Symbolism and the Language of Geo-Architecture (Geo-Architecture and Landscape in China's Geographic and Historic Context Series Volume 4). Singapore: Springer Nature. 